\title{
Purple Acid Phosphatase5 is required for maintaining basal resistance against Pseudomonas syringae in Arabidopsis
}

\author{
Sridhar Ravichandran', Sophia L Stone ${ }^{2}$, Bernhard Benkel ${ }^{3}$ and Balakrishnan Prithiviraj ${ }^{*}$
}

\begin{abstract}
Background: Plants have evolved an array of constitutive and inducible defense strategies to restrict pathogen ingress. However, some pathogens still manage to invade plants and impair growth and productivity. Previous studies have revealed several key regulators of defense responses, and efforts have been made to use this information to develop disease resistant crop plants. These efforts are often hampered by the complexity of defense signaling pathways. To further elucidate the complexity of defense responses, we screened a population of T-DNA mutants in Colombia-0 background that displayed altered defense responses to virulent Pseudomonas syringae pv. tomato DC3000 (Pst DC3000).

Results: In this study, we demonstrated that the Arabidopsis Purple Acid Phosphatse5 (PAP5) gene, induced under prolonged phosphate $\mathrm{Pi}$ ) starvation, is required for maintaining basal resistance to certain pathogens. The expression of PAP5 was distinctly induced only under prolonged Pi starvation and during the early stage of Pst DC3000 infection (6 h.p.i). T-DNA tagged mutant pap5 displayed enhanced susceptibility to the virulent bacterial pathogen Pst DC3000. The pap5 mutation greatly reduced the expression of pathogen inducible gene PR1 compared to wild-type plants. Similarly, other defense related genes including ICS1 and PDF1.2 were impaired in pap5 plants. Moreover, application of BTH (an analog of SA) restored PR1 expression in pap5 plants.

Conclusion: Taken together, our results demonstrate the requirement of PAP5 for maintaining basal resistance against Pst DC3000. Furthermore, our results provide evidence that PAP5 acts upstream of SA accumulation to regulate the expression of other defense responsive genes. We also provide the first experimental evidence indicating the role PAP5 in plant defense responses.
\end{abstract}

Keywords: Arabidopsis, Plant defense responses, PAP5, Pseudomonas syringae, Phosphate starvation

\section{Background}

Plants are continuously exposed to a diverse array of microorganisms including beneficial mutualists, commensals, and pathogens. To defend against pathogens, plants have evolved an innate immune system to recognize and limit infection (reviewed in [1,2]). Activation of defense responses involves the initial recognition of pathogens by chemical cues (elicitors) or Pathogen Associated Molecular Patterns (PAMPs) that include bacterial lipopolysaccharides, flagellin, fungal chitin and ergosterol [3,4]. Recognition of PAMP by specific Pattern Recognition

\footnotetext{
* Correspondence: bprithiviraj@dal.ca

${ }^{1}$ Department of Environmental Sciences, Faculty of Agriculture, Dalhousie University, Truro, NS B2N 5E3, Canada

Full list of author information is available at the end of the article
}

Receptors (PRRs) in the plasma membrane leads to activation of defense responses in both non-host and basal disease resistance [5]. Activation of PRRs subsequently induces the calcium-dependent protein kinase (CDPK) and mitogen-activated protein kinase (MAPK) signaling pathways leading to rapid ion fluxes, followed by transcriptional activation of defense responsive genes and synthesis of antimicrobial compounds to restrict infection [6,7].

Primarily, regulation of plant defense responses is mediated through the phytohormones salicylic acid (SA), jasmonic acid (JA) and ethylene (ET) [8,9]. However, in recent years other phytohormones including abscisic acid (ABA), auxins, gibberellins (GA), cytokines (CK) and brassinosteriods (BR) have been shown to mediate specific plant defense responses (reviewed in $[2,10]$ ). As

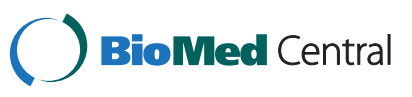


plants are exposed to an array of pathogens with diverse infection strategies, activation of appropriate, pathogenspecific defense responses is vital for plant growth and productivity [11].

Plant pathogens are classified as biotrophs, necrotrophs or hemi-biotrophs based on their life style and infection strategy. Biotrophic pathogens live as obligate parasites that derive nutrients from living host tissues, while necrotrophs feed on dead tissues. Hemi-biotrophs behave as both biotroph and necrotroph depending on the stage of their life cycle [11]. Defense against biotrophs involves SA-dependent responses whereas necrotroph resistance is SA-independent relaying primarily on JA/ET-dependent pathways [9]. The SA signaling pathway is associated with transcriptional activation of pathogenesis related (PR) genes and the establishment of systemic acquired resistance (SAR) to provide enhanced, long lasting resistance to secondary infections $[12,13]$. By contrast, JA/ET signaling pathways are associated with resistance against necrotrophic pathogens and rhizobacteria-mediated induced systemic resistance (ISR), and are not typically associated with PR gene expression $[12,14]$. However, there are complex signaling and cross talk between the SA-dependent and SA-independent pathways [13].

Genetic screening of mutant plant populations has proved very useful for the functional analysis of defense responses [15-17]. In Arabidopsis, genetic screening has revealed a large number of mutants that exhibit altered responses to SA, JA and/or ET and are more susceptible to virulent pathogens [18]. Identification and characterization of enhanced disease susceptibility (eds) mutants, including a series of phytoalexin deficient ( $p a d$ ) mutants, have helped to elucidate a number of defense signaling pathways involved in both basal and induced defense responses [19-21].

Purple Acid Phosphatases (PAPs) belong to a family of binuclear metalloenzymes that exhibit diverse biological functions in plants, animals and bacterial species [22,23]. While the predominant role of PAPs in plants is regulation of Pi uptake, PAPs also contribute to other biological functions including peroxidation [24], ascorbate recycling [25], mediation of salt tolerance [26] and regulation of cell wall carbohydrate biosynthesis [27]. Plant PAPs share significant sequence similarity with mammalian tartarate-resistant acid phosphatases (TRAPs), which are involved in bone resorption [28], iron transport [29] and also in the generation of reactive oxygen species for microbial killing [30]. In humans, TRAP expression is restricted to activated macrophages where it aids in the generation of free radicals to enhance microbial killing [31]. Although numerous reports have emphasized the importance of PAPs in Pi acquisition, it has been difficult to assign a general physiological role to PAPs due to their diversity [32]. The Arabidopsis genome contains 29 PAP encoding genes [33]. Changes in PAP gene expression differs in response to Pi concentration where PAP11 and PAP12 are transcriptionally induced while PAP7-PAP10 and PAP13 remain unchanged in response to Pi deprivation [33]. Kaffarnik and colleagues first reported the accumulation of PAP10 and a decrease in the abundance of PAP14 in the secretome of Arabidopsis cell culture following $P$. syringae infection, suggesting a role for PAPs in the host defense response [34]. Recently, Li et al., (2012) also provided the evidence that some soybean PAPs (GmPAPs) are involved in symbiosis under Pi starved conditions. PAPs carry predicted signal peptides and presumably are secreted, however the biological function of these proteins in the extracellular space is unknown [34].

Here we provide evidence that the Arabidopsis PAP5 is involved in basal resistance against certain plant pathogens. PAP5 mutant plants exhibited enhanced susceptibility to virulent isolate of Pseudomonas syringae pv. tomato DC3000. In addition, expression of defense related genes following Pst DC3000 infection were impaired in pap5 plants.

\section{Results}

Identification of mutants exhibiting altered defense responses One thousand two hundred unique Arabidopsis thaliana (ecotype Col-0) T-DNA insertion lines were spray inoculated with the virulent isolate of Pseudomonas syringae pv. tomato DC3000 (Pst DC3000) and monitored for altered responses to the pathogen. Mutants exhibiting extensive chlorosis in comparison to wild-type plants, scored by visual examination, were designated as susceptible. Mutants exhibiting reduced chlorosis compared to wild type (Col-0) were designated resistant to Pst DC3000. T-DNA insertion lines were also tested for altered root colonization with the plant growth promoting rhizobacterial isolate Pseudomonas putida WCS358. Selected T-DNA lines were retested for response to Pst DC3000. A total of 24 T-DNA insertion lines exhibited either altered disease susceptibility, root colonization or both compared to wild-type plants (data not shown). The mutant line salk_126152C (pap5-1), which exhibited enhanced susceptibility to Pst DC3000 with extensive chlorosis on leaf tissues, was selected for further analysis (Figure 1A). Salk_126152C carried a T-DNA insertion in the gene coding for Purple Acid Phosphatase5 (PAP5; At1G52940) (Genome-Wide Insertional Mutagenesis of Arabidopsis thaliana, 2003). The enhanced susceptibility phenotype of pap5-1 plants was confirmed by assessing bacterial growth in leaf tissues post inoculation. As shown in Figure 1B, pap5-1 plants had greater titers of bacteria at 48 and 72 hours post inoculation (h.p.i) compared to the wild-type plant. To ensure that the altered responses to the pathogen were caused by disruption of 

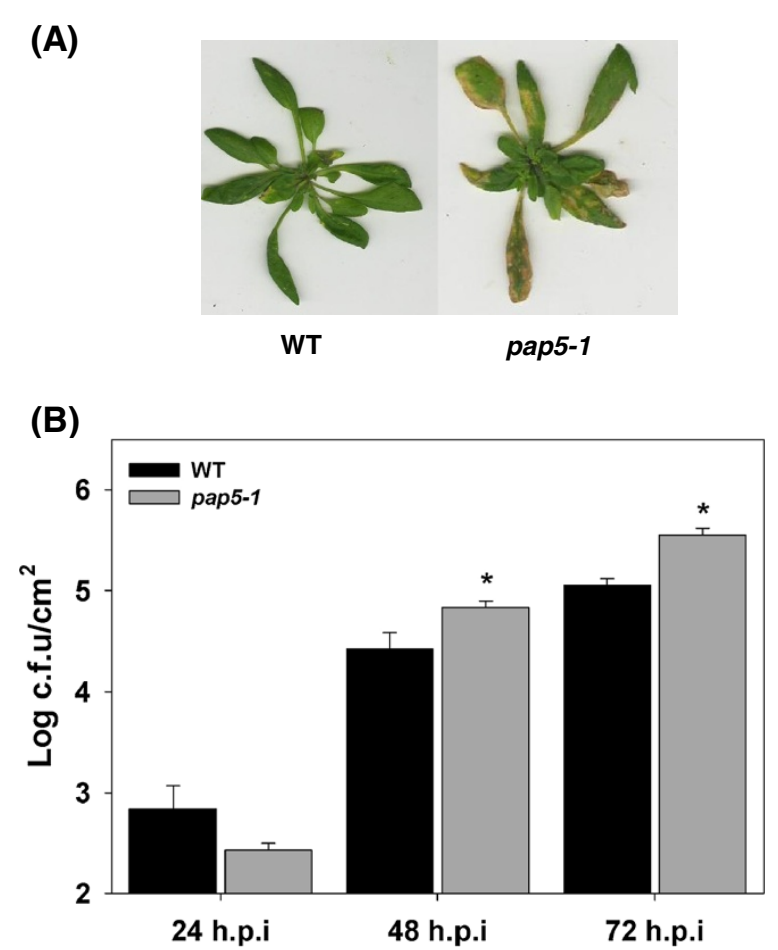

Figure 1 pap5-1 plants exhibit enhanced susceptibility to Pst DC3000. A, Phenotype of pap5-1 plants exhibiting extensive chlorosis and enhanced susceptibility to Pst DC3000. Plants were spray inoculated with $10^{8}$ c.f.u $\mathrm{ml}^{-1}$ and photographed after 5 days of inoculation. B, Growth of virulent Pst DC3000 in wild type (Col-0) and pap5-1 leaves. Plants were spray inoculated with Pst DC3000 (10 c.f.u $\mathrm{ml}^{-1}$ ) and bacterial growth in plant apoplast was determined as described in the materials and methods. The bars represent the mean and standard deviation from values of six to eight replicate samples. The experiment was repeated three times with similar results. An asterisk indicates significance (Student's $t$-test; $P<0.05$ ).

the PAP5 gene and not by an unlinked mutation, a second knockout mutant line salk_081481C (pap5-2), carrying a T-DNA insertion on PAP5 (At1g52940), was tested. pap5-2 plants also exhibited the extensive chlorosis and higher titer of bacteria similar to that of in pap5-1 plants (Additional file 1: Figure S1).

\section{Further characterization of pap5-1 mutant plants}

Genotyping via polymerase chain reaction (PCR) confirmed that pap5-1 (salk_126152C) carries a T-DNA insertion within the first intron (Figure $2 \mathrm{~A}$ and $2 \mathrm{~B}$ ). To determine the impact of T-DNA insertion on transcript levels of PAP5, Reverse Transcription-quantitative PCR (RT-qPCR) was performed using gene specific primers (Figure 2A). Most PAPs are reported to be highly inducible under phosphate starvation $(\mathrm{Pi})$. In our experiments, we did not observe an induction of PAP5 in wild-type seedlings grown in the presence of phosphate (1.25 $\mathrm{mM})$ or under phosphate starved conditions for 5 days $(-\mathrm{Pi}, 0 \mathrm{mM})$ (data not shown). We also observed that the expression of PAP5 under optimal growing conditions was very low and this was confirmed with PAP5 expression profile in the comprehensive microarray site https://www.genevestigator.com/gv/ (Additional file 2: Figure S2). Interestingly, we observed a marked increase in the expression PAP5 when wild type seedlings were grown under prolonged phosphate starvation (Figure $2 \mathrm{C}$ ). For prolonged $\mathrm{Pi}$ starvation wild-type seedlings were germinated in media containing reduced Pi $(0.25 \mathrm{mM})$ for seven days and then transferred to media with no $\mathrm{Pi}$ (0 mM). After 9 days the seedlings were harvested for gene expression analysis. RT-qPCR analysis revealed a $\sim 30$ fold increase in transcript levels of PAP5 in wildtype seedlings grown under prolonged phosphate starvation $(-\mathrm{Pi})$ compared to seedlings grown in the presence of phosphate $(+\mathrm{Pi})$ (Figure $2 \mathrm{C}$ ). The expression of PAP5 was not induced in both pap5-1 (Figure 2C) and pap5-2 (Additional file 3: Figure S3B) seedlings grown under prolonged phosphate starvation (-Pi). We did not observe any major alteration in germination, growth and development of pap5 mutant plants compared to wildtype under optimal growth conditions (data not shown).

\section{Mutation in PAP5 alters expression of host defense responsive genes and ROS production}

To explore the enhanced susceptibility of pap5-1 plants and to determine the role of PAP5 in host defense responses, plants were spray inoculated with virulent isolate of Pst DC3000 $\left(10^{8}\right.$ c.f.u ml $\left.{ }^{-1}\right)$ and the transcript abundances of selected defense responsive genes, including the pathogenesis-related gene1 (PR1), were determined. Infection of wild-type plants with the virulent isolate Pst DC3000 resulted in $\sim 10$-fold induction of the PR1 transcript 24 h.p.i, while an increase of only $\sim 2$-fold was observed in pap5-1 plants (Figure 3). The level of PR1 transcripts in pap5-1 plants following Pst DC3000 infection was variable at 48 h.p.i. However, the expression of PR1 was a still less induced in pap5-1 plants compared to wild-type (Figure 3). Expression of isochorismate synthase1 (ICS1) was induced in wild-type plants ( 2-fold) while no increase in transcript levels was observed in pap5-1 plants. Although, expression of plant defensin1.2 (PDF1.2) was induced ( 2-fold higher) in wild-type plants, expression of PDF1.2 was suppressed in pap5-1 plants (Figure 4A). The expression pattern of these pathogenesis related genes were also confirmed using Actin as the internal control (Additional file 4: Figure S4).

A marked increase in the expression of PAP5 at 6 h.p.i was observed in wild-type plants (Figure 4B). However, this difference did not prolong to 24 and 48 h.p.i. We did not observe induction of PAP5 in mock infected or Pst DC3000 inoculated pap5-1 plants (Figure 4B). The expression profile of PAP5 was further verified from the comprehensive microarray site http://bar.utoronto.ca/ using 


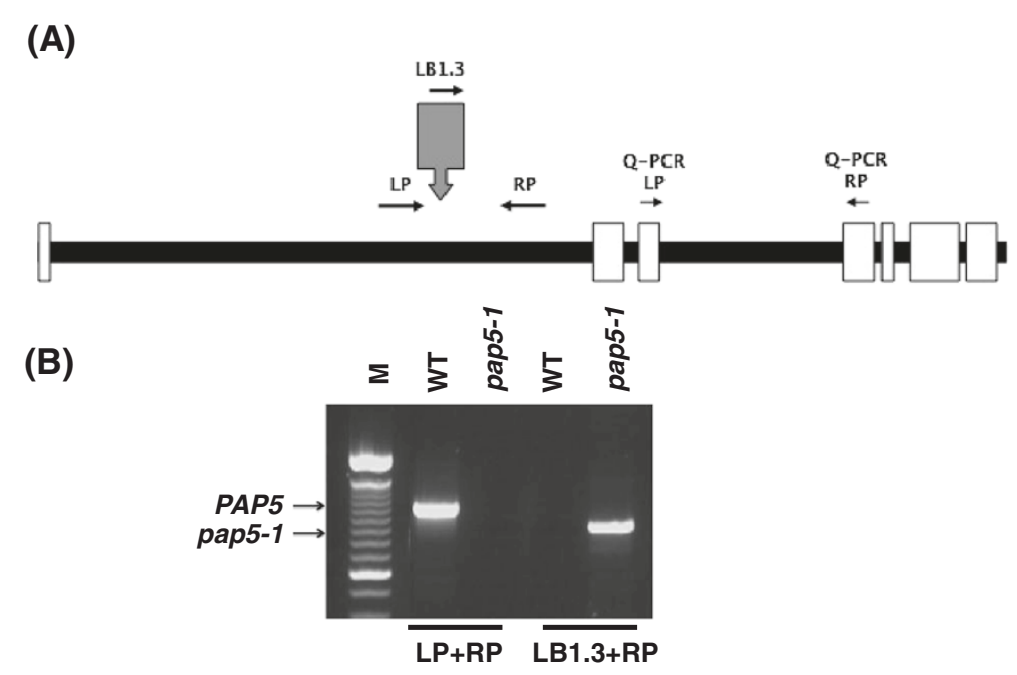

(C)

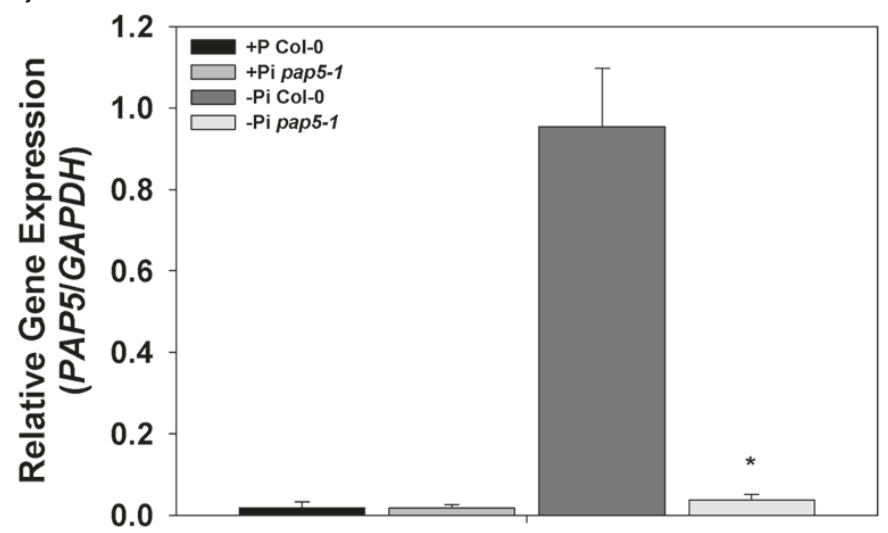

Figure 2 Validation of T-DNA insertion in pap5-1 plants. A, Schematic representation of AtPAP5 (At1G52940); white boxes and solid lines represent exons and introns. T-DNA insertion is represented with a grey arrow and the solid arrows represent the primers used for genotyping and quantitative RT-GPCR. B, Location of the T-DNA insertion and homozygosity of pap5-1 was confirmed by PCR using the gDNA from wild-type and pap5-1 plants (M, 100 bp marker). A 30 cycle PCR reactions was performed with the primer pairs indicated. C, Relative expression of PAP5 transcripts in response to prolonged Pi starvation; For prolonged Pi starvation wild type and pap5-1 seedlings were germinated and grown in 0.5X MS media containing reduced $\mathrm{Pi}(0.25 \mathrm{mM})$. After seven days the seedlings were washed with sterile water and transferred to $0.5 \mathrm{X}$ MS with no Pi (0 mM). After 9 days the seedlings were harvested for gene expression analysis. Total RNA was extracted from wild-type and pap5-1 plants as described in Methods. Transcript levels of PAP5 was normalized to the expression of GAPDH in the same samples and expressed relative to the normalized transcript levels of Pi starved wild-type plants. The bars represent the mean and standard deviation from two independent experiments. Asterisks represents data sets significantly different from the wild-type data sets $(P<0.05$ using one-tailed Student's $t$-test).

Arabidopsis eFP Browser (Additional file 5: Figure S5) [35]. Although, PAP5 was strongly induced only at 6 h.p.i, our results suggest that this level of PAP5 is required for maintaining resistance against virulent Pst DC3000.

To further explore the mechanism of enhanced susceptibility, we studied hydrogen peroxide $\left(\mathrm{H}_{2} \mathrm{O}_{2}\right)$ accumulation using 3-3'-Diaminobenzidine (DAB) staining. As shown in Figure 5A, accumulation of $\mathrm{H}_{2} \mathrm{O}_{2}$ in response to Pst DC3000 was reduced in pap5-1 leaves at 24 and 48 h.p.i. In contrast, there was an accumulation of $\mathrm{H}_{2} \mathrm{O}_{2}$ in the wild-type plants. The $\mathrm{H}_{2} \mathrm{O}_{2}$ concentration was quantified in leaf tissues following Pst DC3000 infection. The wild-type plants accumulated a higher concentration of $\mathrm{H}_{2} \mathrm{O}_{2}$ in response to Pst DC3000 inoculation as compared to pap5-1 plants (Figure 5B).

\section{Resistance to Botrytis cinerea is affected in pap5 plants}

Having demonstrated the enhanced susceptibility of pap5-1 plants to the hemi-biotrophic pathogen Pst DC3000, we next tested the level of resistance of pap5-1 plants to the necrotrophic pathogen Botrytis cinerea. Four week old plants were inoculated with spore suspension of $B$. cinerea and lesion size was measured three days later. As shown in Figure 6A, pap5-1 plants developed a significantly larger lesion $(5.4 \pm 0.3 \mathrm{~mm})$ than the wild-type $(3.9 \pm 0.2 \mathrm{~mm})$. The greater lesion size on 


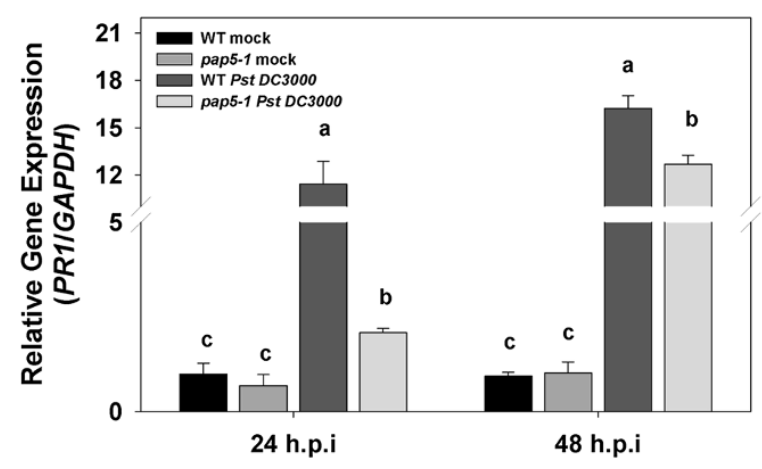

Figure 3 Expression of $P R 1$ in wild-type and pap5-1 plants after Pst DC3000 infection. Transcript levels of $P R 1$ in wild-type and pap5-1 plants were quantified after spray inoculation with virulent

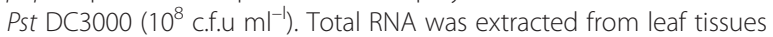
sampled 24 and 48 h.p.i. Transcript levels were normalized to the expression of GAPDH in the same samples. The transcript levels were expressed relative to the normalized transcript levels of mock

infected wild-type plants. The bars represent the mean and standard deviation from two independent experiments. Significant differences $(P<0.05)$ are indicated by different letters.

pap5-1 plants in response to $B$. cinerea infection, suggests the role of PAP5 are important in limiting fungal growth.

To identify the role of PAP5 in the resistance against $B$. cinerea, we assessed the transcript abundance of $P R 1$ and PDF1.2. As shown in Figure 7A, B. cinerea strongly induced the expression of PR1 in both wild-type and pap5. In contrast, the level of the PDF1.2 transcript at 24 h.p.i was only half of that observed in wild-type plants (Figure 7B). By 48 h.p.i., however, the transcript levels of PDF1.2 were similar in both wild-type and pap5-1 plants. Similarly, we did not observe any significant differences in PAP5 transcripts with $B$. cinerea infection (Figure 6B).

\section{Responses to exogenous application of BTH, a salicylic acid analog and methyl jasmonate (MJ) is unaffected in pap5 plants}

Since pap5-1 plants exhibited enhanced susceptibility to Pst DC3000 and B. cinerea, we investigated the role of PAP5 in responses to BTH and MJ. Exogenous application of $\mathrm{BTH}$ induced higher levels of $P R 1$ in wild-type and pap5-1 (Figure 8A). We also observed a slightly higher increase in the expression PR1 in pap5-1 plants $24 \mathrm{~h}$ after BTH treatment. Similarly, application of MJ strongly induced the expression of PDF1.2 in both wild-type and pap5-1 plants. We did not observe significant differences in expression of PDF1.2 between wild-type and pap5-1 plants following application of MJ (Figure 8B). Application of BTH and JA induced expression of PR1 and PDF1.2, respectively, indicative of an intact JA signaling pathway in pap5 plants. Based on these experiments it was clear that

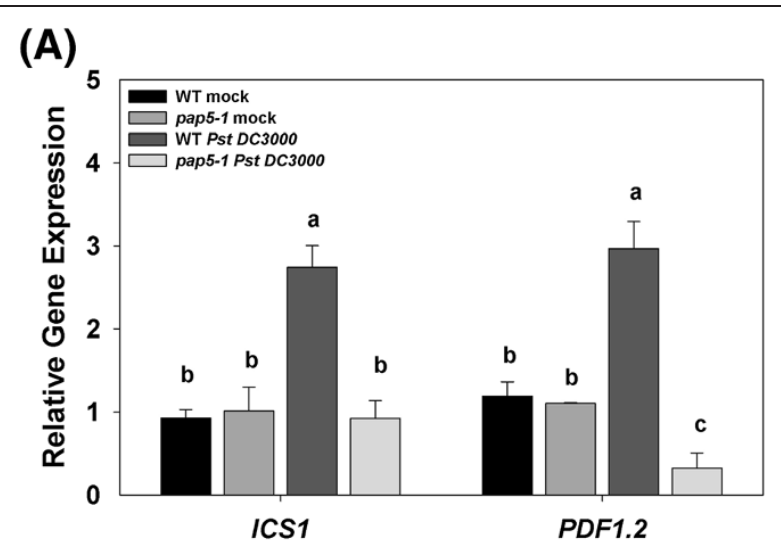

(B)

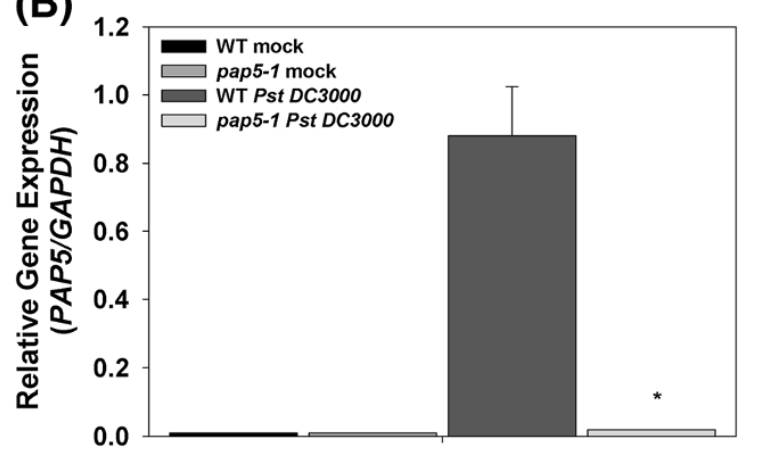

Figure 4 Expression of ICS1, PDF1.2 and PAP5 in wild-type and pap5-1 plants after Pst DC3000 infection. Transcript levels of ICS1, PDF1.2 and PAP5 in wild-type and pap5-1 plants were quantified after spray inoculation with virulent Pst DC3000 (10 c.f.u ml $\left.{ }^{-1}\right)$. A, Expression ICS1 and PDF1.2 following Pst DC3000 infection. Total RNA was extracted from leaf tissues sampled at 24 h.p.i. Transcript levels were normalized to the expression of GAPDH in the same samples. The transcript levels were expressed relative to mock infected wild-type plants. B, Expression of PAP5 following Pst DC3000 infection. Total RNA was extracted from leaf tissues 6 h.p.i. Transcript levels were normalized to the expression of GAPDH in the same samples and expressed relative to transcript levels of infected wild-type plants. The bars represent the mean and standard deviation from two independent experiments. Significant differences $(P<0.05)$ are indicated by different letters. Asterisks indicate significant difference in transcript levels compared to wild-type (Students $t$-test; $P<0.05$ ).

pap5-1 plant was not defective in responding to exogenously applied BTH or MJ.

\section{Discussion}

In this study, we demonstrated the role of PAP5, a phosphate responsive gene, and its requirement in maintaining basal disease resistance against virulent Pst DC3000. In previous studies PAP5 transcripts were not detectable under phosphate starvation [36]. Unlike PAP12 and PAP26, PAP5 is not abundantly expressed under normal phosphate starvation conditions. Our results revealed that PAP5 is expressed only under prolonged $\mathrm{Pi}$ starvation (Figure 2C). Mutation in PAP26 has been shown to impair 


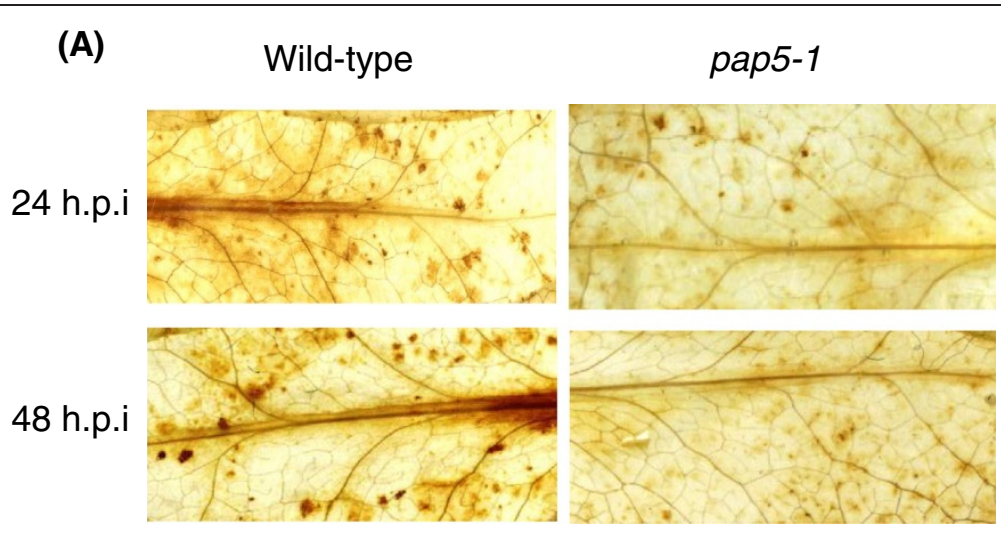

(B)

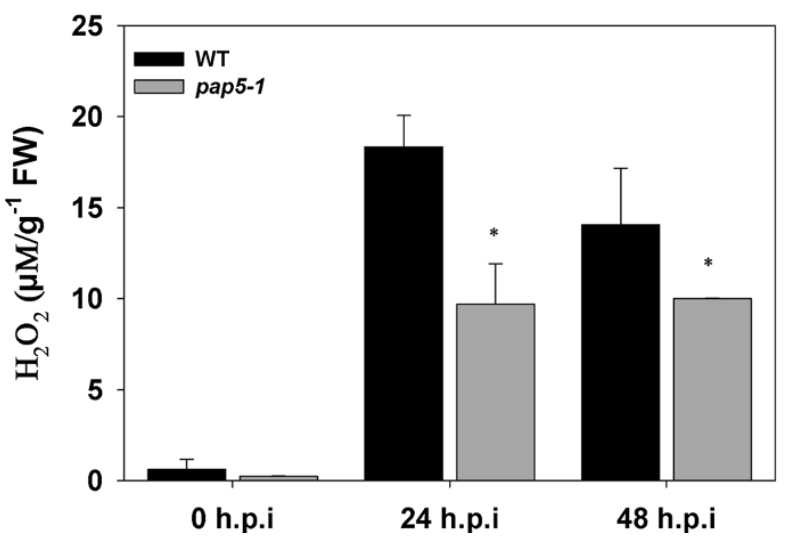

Figure 5 pap5-1 plants accumulate reduced $\mathrm{H}_{2} \mathrm{O}_{2}$ in response to Pst DC3000 infection. A, Histochemical detection of $\mathrm{H}_{2} \mathrm{O}_{2}$ accumulation using DAB staining. Wild-type and pap5-1 leaves were excised following Pst DC3000 infection and stained with DAB for hydrogen peroxide. B, Quantification of $\mathrm{H}_{2} \mathrm{O}_{2}$ following Pst DC3000 infection. The bars represent mean and SD of $\mathrm{H}_{2} \mathrm{O}_{2}$ accumulation. Asterisks represent significant difference in $\mathrm{H}_{2} \mathrm{O}_{2}$ production compared to wild type (Student's $t$-test; $P<0.05$ ).

growth and increase anthocyanin accumulation in response to Pi starvation [37]. Despite the loss of PAP5 expression, mutant plants did not show discrete phenotypic differences from that of wild-type plants. Both wild-type and pap5-1 plants exhibited an increased root/shoot ratio under Pi starvation (data not shown). This finding also indicates that PAP5 does not play a major role in Pi acquisition and is more likely to regulate other functions. The Arabidopsis genome contains 29 PAP encoding genes [33] and this may lead to functional redundancy. This study suggests that the loss of PAP5 resulted in impairment of defense responsive genes in response to Pst DC3000 infection. Further, it appears that other PAP genes does not compensate for the loss of PAP5 function in response to pathogen attack.

Genetic analyses of Arabidopsis mutants have revealed many key regulatory genes in plant defense responses. Enhanced disease susceptibility mutants including eds5, pad4, npr 1 and sid2 have previously been reported to exhibit enhanced susceptibility and compromised defense responses to both virulent and avirulent isolates of Pst
DC3000 [19,20,38]. It is also evident that most bacterial pathogens including Pst DC3000 are inoculated by pressure-infiltration to study plant-bacterial interactions. Although pressure-infiltration is the most commonly used inoculation method, these inoculation procedures may prevent early innate immune responses such as flagellin perception (FLS2 mediated resistance) [39] and stomatal closure [40]. Also FLS2 mediated resistance was effective only when Pst DC3000 was sprayed on the leaf surface and not when bacteria was infiltrated in to leaves [39]. Hence, to mimic natural infection and to focus on the early defense responses we sprayed plants with suspension of Pst DC3000 containing $10^{8}$ c.f.u ml ${ }^{-1}$. We also observed that plants sprayed with $10^{3}$ and $10^{5}$ cells $/ \mathrm{ml}^{-1}$ developed reduced symptoms compared to plants sprayed with $10^{8}$ cells (data not shown). Similar bacterial titers have been previously used for plant-bacterial interaction studies $[39,40]$.

We observed that the expression of $P R 1$ was slightly induced in pap5-1 plants following Pst DC3000 infection, however the relative transcript level of $P R 1$ was 


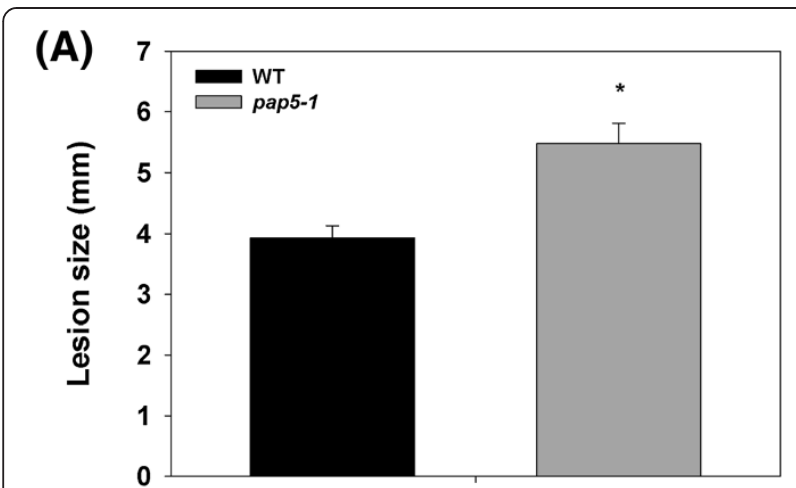

(B)

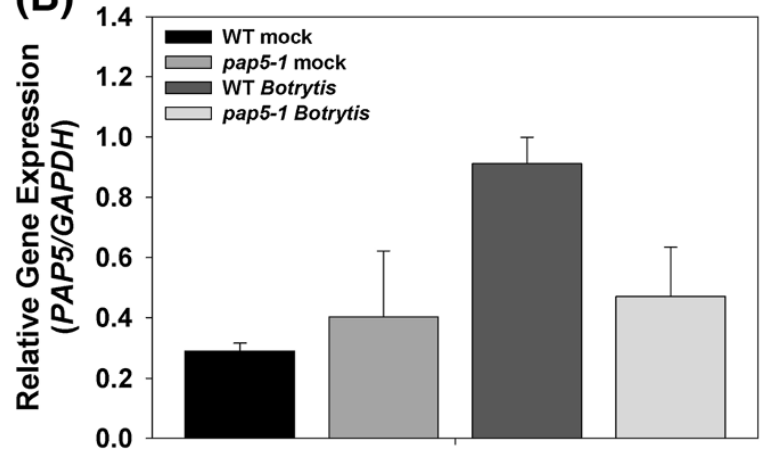

Figure 6 Lesion development and induction of PAP5 following Botrytis cinerea infection. A, Size of lesion in wild type and pap5-1 plants inoculated after $B$. cinerea infection. Leaves were inoculated by placing $5 \mu$ l of the $B$. cinerea spore suspension $\left(1 \times 10^{5} / \mathrm{ml}\right)$ on either side of the mid vein and the lesion size was measured after 3 days. The bars represent mean and SD of 20 individual lesions. Asterisks represent significant difference in lesion size compared to wild-type (Student's t-test; $P<0.05)$. B, PAP5 expression in response to $B$. cinerea infection. Leaf tissues were harvested 48 h.p.i for RNA extraction. Transcript levels of PAP5 were normalized to the expression of GAPDH in the same samples. The transcript levels were expressed relative to the normalized transcript levels of infected wild-type plants.

several fold lower compared to wild-type (Figure 3 ). The PR1 transcripts at 48 h.p.i were slightly lower compared to wild-type (Figure 3). Similar variability has been observed in MPK6 silenced plants that were susceptible to Pst DC3000 [41]. We observed that PAP5 was strongly induced in the early stages of infection (6 h.p.i). This induction was transient as no difference was observed at 24 and 48 h.p.i. One possible explanation of this observation is that the level of PAP5 induced during the early stages (6 h.p.i) of infection could be sufficient to dephosphorylate signaling proteins required for activation of defense responses downstream of PAP5. Thus, it is also possible that PAP5 might be involved in early responses to pathogens similar to glutotione s-transferse (GST6) and glucosyltransferase [42]. Moreover, members of the PAP family have been known to exhibit peroxidase activity in addition to Pi acquisition and recycling $[24,43]$.
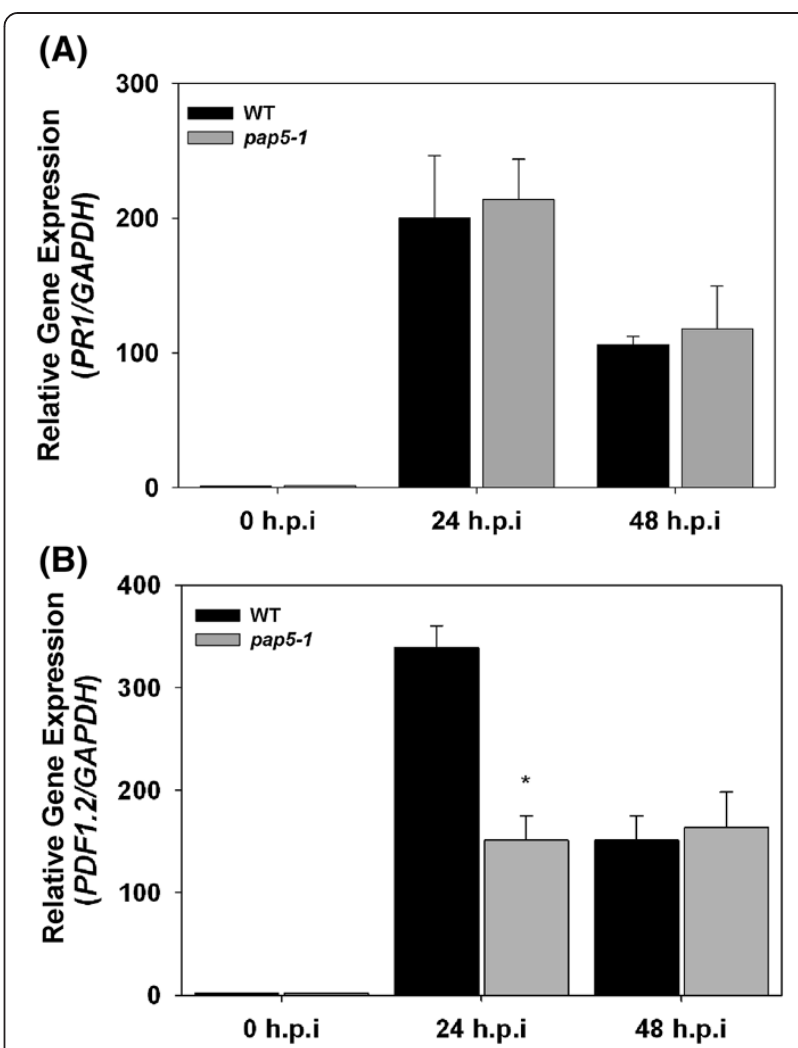

Figure 7 Induction of $P R 1$ and PDF1.2 in response to Botrytis cinerea. $\mathbf{A}$, Expression $P R 1$ in response to $B$. cinerea infection.

$\mathbf{B}$, expression of PDF1.2 in response to $B$. cinerea infection. Plants were spray inoculated with spore suspension of $B$. cinerea $\left(1 \times 10^{5}\right)$ and leaf tissues were harvested for total RNA extraction. Transcript levels of PR1 and PDF1.2 was normalized to the expression of GAPDH in the same samples and expressed relative to the normalized transcript levels of mock infected wild-type plants. The bars represent the mean and standard deviation from two independent experiments. Asterisks indicate significant difference in transcript levels compared to wild-type (Students $t$-test; $P<0.05$ ). Induction of $P R 1$ and PDF1.2 in response to Botrytis cinerea infection.

Although, the role of PAP5 with regard to peroxidase activity has not been established, we hypothesize that the PAP5 might mediate generation of reactive oxygen species (ROS) during Pst DC3000 infection. ROS was initially proposed to be mediate plant defense response especially, during an incompatible interaction [44]. Virulent pathogens, capable of evading pathogen recognition are also known to induce ROS production at latter stages of infection to lower levels [1].

We also identified the importance in PAP5 in limiting the growth of the necrotrophic fungus, $B$. cinerea at the site of infection. The expression of PDF1.2 was strongly suppressed in pap5-1 plants at 24 h.p.i resulting in an increase in lesion size. There were no differences in PDF1.2 transcripts between pap5 and wild-type plants at 48 h.p.i. Similarly, eds4-1 plants have been reported to exhibit enhanced susceptibility to $B$. cinerea despite 

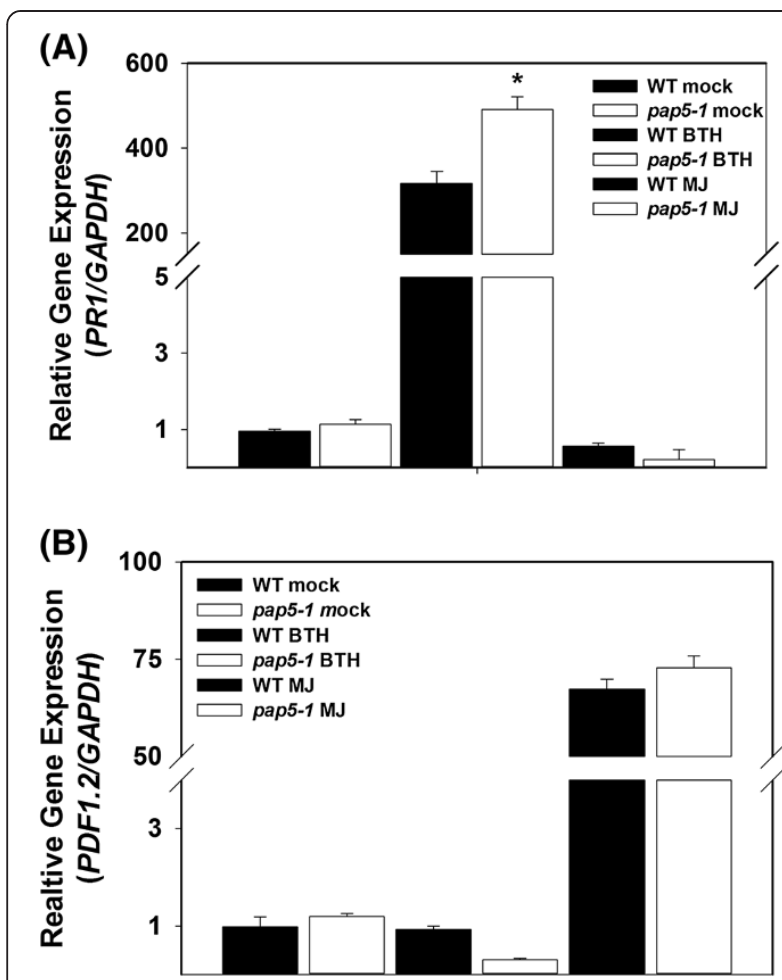

Figure 8 Induction of $P R 1$ and PDF1.2 following exogenous application of benzothiozidole and methyl jasmonate.

A, Expression PR1 in response to benzothiozidole (BTH) treatment. B, expression of PDF1.2 in response to methyl jasmonate treatment. Plants were spray treated with either or $0.06 \%$ of Actigard ${ }^{\circledR}$ (Active ingradient: 50\% w/w benzothiozidole) or $50 \mu \mathrm{M}$ methyl jasmonate. Leaf tissues were harvested after 24 of spraying for total RNA extraction. Transcript levels of PDF1.2 and PR1 were normalized to the expression of GAPDH in the same samples and expressed relative to the normalized transcript levels of mock treated wild-type plants. The bars represent the mean and standard deviation from two independent experiments. Asterisks indicate significant difference in transcript levels compared to wild-type (Students $t$-test; $P<0.05$ ).

comparable expression of $P R 1$ and $P D F 1.2$ transcripts [45]. These results also suggest that defense responsive genes other than PR1 and PDF1.2 are required to mount wild-type levels of resistance against $B$. cinerea. SA synthesized in response to $B$. cinerea infection has reported to be derived via phenylalanine ammonia lyase $(P A L)$ and not via isochorismate synthase (ICS) [46]. Since pap5-1 plants induced comparable levels of $P R 1$ to wildtype plants following $B$. cinerea infection, it is possible that the effect of PAP5 is restricted to SA derived via ICS and not via PAL.

Application of BTH and MJ in wild-type and pap5 plants induced expression of $P R 1$ and PDF1.2, respectively (Figure $8 \mathrm{~A}$ and $8 \mathrm{~B}$ ). These results also suggest that PAP5 is not required for expression of SA dependent PR1 expression. PR1 expression in pap5-1 plants appeared to be slightly higher than wild-type plants after of BTH treatment (Figure 8A). This slight increase in PR1 expression and its significance is unclear. Similarly, application of SA on pad4 plants showed a slight increase in PR1 expression [47]. Application of MJ induced the expression of $P D F 1.2$, indicating the regulatory function of PAP5 to be upstream of SA and JA.

Although, most plant PAPs are primarily associated with Pi absorption and recycling, PAPs induced under Pi starvation are also known to exhibit peroxidase activity similar to mammalian PAPs $[24,48]$. All mammalian PAPs characterized exist as monomers of $\sim 35 \mathrm{kDa}$ (Low Molecular Weight, LMW), while plants encode a relatively large family of High Molecular Weight (HMW) homodimeric and oligomeric PAPs ( 45-74 kDa). However, a recent study has identified mammalian-like low molecular weight PAP $(\sim 34 \mathrm{kDa})$ from roots of Pi starved bean plants [32]. Moreover, the LMW, $35 \mathrm{kDa}$ plant PAPs are reported to be closely related to the $35 \mathrm{kDa}$ mammalian PAPs than to the large plant PAPs [49]. Thus, from our results we hypothesize that PAP5 could play a role in both Pi acquisition and in microbial killing during pathogenesis (Figure 9).

\section{Conclusion}

We identified the requirement of PAP5 for maintaining basal defense responses against virulent Pst DC3000, suggesting a role for PAP5 in pathogen triggered immunity (PTI). We further demonstrated that PAP5 acts upstream of SA to affect the expression of $P R 1$, and levels of PAP5 do not affect BTH and JA perception. Further analysis on pap5 plants is likely to reveal novel components of signal transduction pathways that regulate defense responsive genes.

\section{Methods}

\section{Biological materials and growth conditions}

Arabidopsis thaliana (L.) Heynh, ecotype Columbia (Col-0) seed was purchased from Lehle seeds (Round Rock, TX, USA) and T-DNA insertion mutant lines were obtained from Arabidopsis Biological Resource Center (Columbus, $\mathrm{OH}, \mathrm{USA})$. Seeds were surface sterilized with $\mathrm{NaOCl} 2 \%$ $(\mathrm{v} / \mathrm{v})$, rinsed five times with sterile water and stratified at $4^{\circ} \mathrm{C}$ for 3 days. Seeds were planted either in Jiffy peat pellets (Halifax seeds, Canada) or on plates with 0.5X MS media [50]. Plants were grown at $22 \pm 2^{\circ} \mathrm{C}$ with a photoperiod of $16 \mathrm{~h}$ light at $125 \mu \mathrm{mol} \mathrm{m} \mathrm{s}^{-2}$ and $8 \mathrm{~h}$ dark cycle.

Virulent Pseudomonas syringae pv. tomato DC3000 (Pst DC3000) was kindly gifted by Dr. Diane Cuppels, Agriculture and Agri Food Canada (AAFC), ON, Canada. Pseudomonas syringae strain was maintained on King's medium B supplemented with rifampicin $\left(50 \mu \mathrm{g} \mathrm{ml}^{-1}\right)$. Botrytis cinerea was cultured on modified King's medium B (10 g peptone, $1.5 \mathrm{~g}$ potassium phosphate monobasic, $15 \mathrm{~g}$ dextrose, $\mathrm{pH} 5.5,5 \mathrm{ml}$ of $1 \mathrm{M} \mathrm{MgSO} / \mathrm{l})$. 


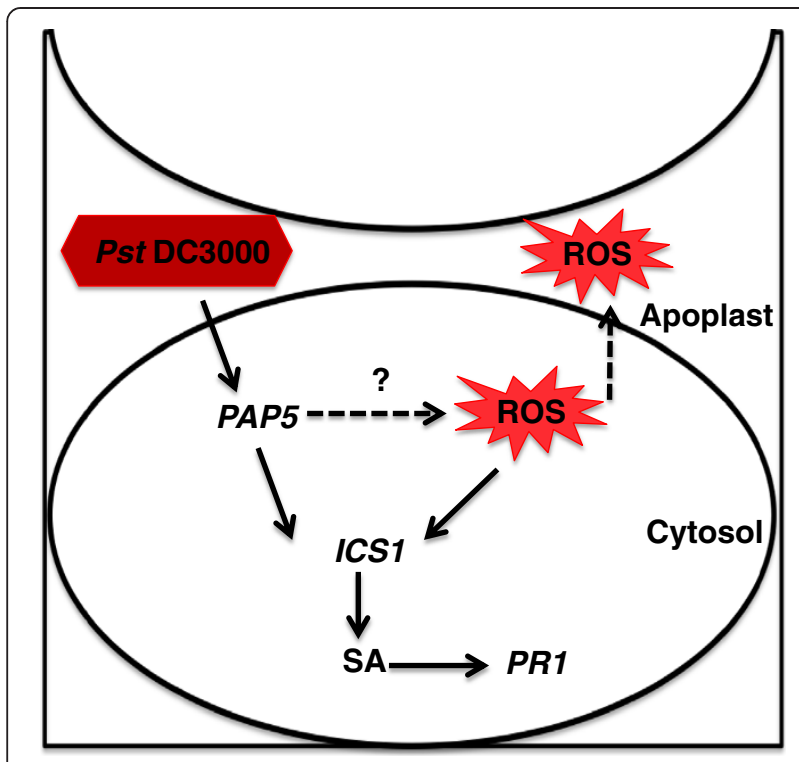

Figure 9 Model for role of PAP5 during Pst DC3000 infection. When plants are infected with virulent Pst DC3000, PAP5 is required for activation of defense responsive genes including PRI and ICSI. Recognition of Pst DC3000, induce expression of PAP5 only during the early stages of infection ( $6 \mathrm{~h}$ ) and triggers ROS synthesis which subsequently activates other defense related signals down stream for complete resistance.

For plant treatment, Benzothiozidole (Actigard ${ }^{\oplus}$; active ingradient $50 \% \mathrm{w} / \mathrm{v}$ BTH) was a gift from Syngenta Corp., USA. Methyl jasmonate and other microbiological media were purchased from Sigma Aldrich, Oakville, Canada.

\section{Mutant screening and pathogen inoculation}

Genetic screen was performed on 4 to 5 week old plants by spray inoculation with bacterial suspension of virulent Pst DC3000. Plant inoculation and bacterial growth in plant apoplast was determined as described by [39]. In brief, strains of virulent Pseudomonas syringae pv. tomato DC3000 (Pst DC3000) was cultured in King's medium B supplemented with rifampicin $\left(50 \mu \mathrm{g} \mathrm{ml}^{-1}\right)$ at $28^{\circ} \mathrm{C}$ until $\mathrm{OD}_{600}$ of 0.8 . Bacterial cells were collected by centrifugation and resuspended in water containing 0.02\% Silwet L-77 (Lehle seeds, USA) to a final concentration of $10^{8}$ c.f.u ml ${ }^{-1}$. Plants (4-5 weeks old) were spray inoculated and kept under high humidity for disease development. Leaves were excised (8-10 replicates) from different infected plants and were surface sterilized with ethanol $(75 \% \mathrm{v} / \mathrm{v})$. Four to five samples were made by pooling 2 leaf discs $\left(0.5 \mathrm{~cm}^{2}\right)$ and the samples were ground in sterile water with microfuge tube pestle. The ground tissues were serially diluted and plated on King's B medium containing rifampicin $(50 \mu \mathrm{g} / \mathrm{ml})$. The plates were incubated at $28^{\circ} \mathrm{C}$ and colonies were counter after 48 hours. For Pst DC3000 induced gene expression, plants were spray inoculated with bacterial suspension $\left(10^{8}\right.$ c.f.u $\left.\mathrm{ml}^{-1}\right)$ and leaf tissues were frozen in liquid nitrogen at the time points indicated.

For Botrytis cinerea $(\mathrm{Bcr})$ inoculation, spore suspension $\left(1 \times 10^{5}\right.$ conidia $\left.\mathrm{mL}^{-1}\right)$ was prepared in potato dextrose broth (PDB) as described by [45]. Four to five week old plants were inoculated by placing $5 \mu$ l of the spore suspension on either side of the mid vein of fully expanded leaves. Inoculated plants were covered with a transparent plastic dome to maintain high humidity for disease development. For all gene expression analysis, leaf tissues were harvested from four individual plants for each biological replicate and were snap frozen in liquid nitrogen for RNA extraction.

Benzothiozidole (BTH) and methyl jasmonate (MJ) treatments were performed by spraying 4-5 weeks old plants with solutions containing $0.06 \% \mathrm{w} / \mathrm{v}$ Actigard ${ }^{\circ}$ (Active ingradient: $50 \% \mathrm{w} / \mathrm{v} \mathrm{BTH}$ ) or $50 \mu \mathrm{M}$ methyl jasmonate (MJ) with $0.02 \%$ Silwet L-77.

\section{Confirmation of T-DNA insertion}

T-DNA insertion and homozygosity of mutant line salk_126152 was confirmed by PCR as described by [51] using AtPAP5 gene specific primers generated from SALK T-DNA verification primer design tool LP 5'TTCACGGTTTTGTTGTTAGACG-3', RP 5'-TCGTTG AAAACTACACTCGATTTAAC-3' and left border primer LBb1.3 5'-ATTTTGCCGATTTCGGAAC-3'.

\section{Phosphate starvation}

Sterile, stratified seeds (20-25 per jar) were dispensed in $50 \mathrm{ml}$ of liquid $0.5 \mathrm{X}$ MS medium containing $\mathrm{Pi}$ (1.25 mM) or with reduced $\mathrm{Pi}(0.25 \mathrm{mM})$. The seedlings were grown under constant shaking $(85 \mathrm{rpm})$ at $22 \pm 2^{\circ} \mathrm{C}$ under continuous illumination at $100 \mu \mathrm{mol} \mathrm{m} \mathrm{m}^{-2} \mathrm{~s}^{-1}$. After 9 days the seedlings were rinsed thrice with sterile distilled water and transferred to $0.5 \mathrm{X}$ MS medium containing + $\mathrm{Pi}(1.25 \mathrm{mM})$ or $-\mathrm{Pi}(0 \mathrm{mM})$ [52]. Plants were harvested after 11 days for RNA extraction.

Table 1 Primer sequences used in RT-qPCR experiments

\begin{tabular}{lll}
\hline Gene & Locus & Primer sequences (5' $^{\prime}$ - ${ }^{\prime}$ ) \\
\hline GAPDH & At1g13440 & TTGGTACAACAGGTCAAGCA \\
& AAACTTGTCGCTCAATGCAATC \\
ICS1 & At1g74710 & GCGTCGTTCGGTACAGG \\
& & ACAGCGAGGCTGAATATCAT \\
PAP5 & At1g52940 & AACAGGTCGCTCCACTAGACA \\
& & TGGTAGAGGCATATGTTGTCC \\
PDF1.2 & At5g44420 & GTCTCTTGCTGCTTCGAC \\
& & GCAAACCCCTGACCATGT \\
PR1 & At2g14610 & TGATCCTCGTGGGAATATGT \\
& & TGCATGATCACATCATTACTTCAT \\
\hline
\end{tabular}


Whenever Pi was reduced from growth medium, equivalent amounts of sulphate salts were added to maintain the concentration of conjugate cations.

\section{RNA extraction and quantitative Real-time PCR}

Total RNA was extracted from frozen tissues using monophasic extraction method [53]. Reverse Transcription was performed with $2 \mu \mathrm{g}$ of total RNA using Quantiscript RTase (Qiagen, ON, Canada). Relative transcript levels were assayed by Real-Time PCR using gene specific primers (Table 1) on StepOnePlus Real-Time PCR system (Applied Biosystems, ON, Canada) using SYBR Green reagent (Applied Biosystems, ON, Canada). To determine the relative expression levels, the amount of target gene was normalized over the abundance of constitutive Glyceraldehyde 3-phosphate dehydrogenase (GAPDH) or Actin as endogenous control. Primers were generated using the Roche Universal Probe Library assay design center.

\section{DAB staining}

To visualize $\mathrm{H}_{2} \mathrm{O}_{2}$ production in situ, plants were inoculated with suspension of Pst DC3000 as described in earlier section. Leaves were excised at 24 and 48 h.p.i and stained with 3-3 Diaminobenzidine (DAB) as described by [54]. Excised leaved were placed in DAB (1 $\mathrm{mg} / \mathrm{ml}$ ) solution for 8-12 hours and the tissues were soaked in ethanol $(95 \%, \mathrm{v} / \mathrm{v})$ to remove chlorophyll. For $\mathrm{H}_{2} \mathrm{O}_{2}$ quantification, the excised leaf tissues were frozen and ground with liquid nitrogen. To $50 \mathrm{mg}$ of ground frozen tissue $500 \mu \mathrm{l}$ of phosphate buffer $(50 \mathrm{mM}$, sodium phosphate, $\mathrm{pH}-7.4$ ) was added. The samples were centrifuged and $50 \mu \mathrm{l}$ of the aliquot was used for $\mathrm{H}_{2} \mathrm{O}_{2}$ quantification using an Amplex red hydrogen peroxide/ peroxidase assay kit (Molecular Probes, Life Technologies, Canada).

\section{Additional files}

Additional file 1: Figure S1. Enhanced susceptibility of pap5-2 to Pst DC3000. A, Phenotype of pap5-2 plants exhibiting extensive chlorosis. Plants were spray inoculated with $10^{8} \mathrm{c.f.u} \mathrm{ml}^{-1}$ and photographed after 5 days of infection. B, Growth of virulent Pst DC3000 in wild type (Col-0) and pap5-2 mutant leaves. Plants were spray inoculated with Pst DC3000 $\left(10^{8}\right.$ c.f.u mll $\left.{ }^{-1}\right)$ and bacterial growth in plant apoplast was determined. The bars represent the mean and standard deviation from values of six to eight replicate samples and the experiment was repeated two times with similar results. An asterisk indicates significant increase in Pst DC3000 growth compared to wild-type (Student's $t$-test; $P<0.05$ ).

Additional file 2: Figure S2. Expression profile of PAP5 (array element 261341_s_at) in comparison to PR1 (array element 266385_at) from Genevestigator Expression Data.

Additional file 3: Figure S3. Validation of T-DNA insertion in pap5-2 mutant plants. A, Schematic representation of AtPAP5 (At1G52940); white boxes and solid lines represent exons and introns. T-DNA insertion is represented with a grey arrow and the solid arrows represent the primers used for genotyping and quantitative RT-qPCR. B, Location of the T-DNA insertion and homozygosity of pap5-2 was confirmed by PCR using the
gDNA from wild-type and pap5-2 plants (M, 100 bp marker). A 30 cycle PCR reactions was performed with the primer pairs indicated. C, Relative expression of PAP5 transcripts in response to Pi starvation; Total RNA was extracted from wild-type and pap5 plants as described in materials and methods. Transcript levels of PAP5 was normalized to the expression of GAPDH in the same samples and expressed relative to the normalized transcript levels of Pi supplemented wild-type plants. The bars represent the mean and standard deviation from two independent experiments. Asterisks represents data sets significantly different from the wild-type data sets $(P<0.05$ using one-tailed Student's $t$-test).

Additional file 4: Figure S4. Expression of defense related genes in wild-type and pap5-1 mutant plants after Pst DC3000 infection. Transcript levels of PR1, ICS1, PDF1.2 and PAP5 in wild-type and pap5-1 plants were quantified after spray inoculation with virulent Pst DC3000 $\left.\left(10^{8} \text { c.f.u ml}\right)^{-1}\right)$ was determined. Total RNA was extracted from leaf tissues harvested 24 h.p.i. Transcript levels were normalized to the expression of Actin in the same samples. The transcript levels were expressed relative to the normalized transcript levels of mock infected wild-type plants. The bars represent the mean and standard deviation. Significant differences $(P<0.05)$ are indicated by different letters.

Additional file 5: Figure S5. Expression profile of PAP5 (At1g52940) from the Arabidopsis eFP Browser.

\section{Competing interests}

The authors declare that they have no competing interests.

\section{Authors' contributions}

BP conceived the concept of genetic screening and designed all experiments. SR carried out all the experiments and prepared the manuscript. SLS critically evaluated all the experiments and significantly contributed to the manuscript preparation. BB helped with sequencing and contributed to evaluate the manuscript. All authors read and approved the final manuscript.

\section{Acknowledgements}

We would like to thank Ms. Kalyani Prithiviraj (Faculty of Agriculture, Dalhousie University) for her assistance with Real-Time PCR analysis and Dr. Hongxia Liu (Department of Biology, Dalhousie University) for her help and advice with our experiments. This work was supported by Natural Science and Engineering Research Council of Canada Discovery grant to B. Prithiviraj.

\section{Author details}

'Department of Environmental Sciences, Faculty of Agriculture, Dalhousie University, Truro, NS B2N 5E3, Canada. ${ }^{2}$ Department of Biology, Dalhousie University, Halifax, NS B3H 4J1, Canada. ${ }^{3}$ Department of Plant and Animal Sciences, Faculty of Agriculture, Dalhousie University, Truro, NS B2N 5E3, Canada.

Received: 15 February 2013 Accepted: 24 July 2013 Published: 29 July 2013

\section{References}

1. Jones JDG, Dangl JL: The plant immune system. Nature 2006, 444(7117):323-329.

2. Pieterse CMJ, Leon-Reyes A, Van dE, Van Wees SCM: Networking by smallmolecule hormones in plant immunity. Nat Chem Biol 2009, 5(5):308-316.

3. Zipfel C, Felix G: Plants and animals: a different taste for microbes? Curr Opin Plant Biol 2005, 8(4):353-360.

4. Boller T, Felix G: A renaissance of elicitors: perception of microbe-associated molecular patterns and danger signals by pattern-recognition receptors. Annu Rev Plant Biol 2009, 60(1):379-406.

5. Bittel P, Robatzek S: Microbe-associated molecular patterns (MAMPs) probe plant immunity. Curr Opin Plant Biol 2007, 10(4):335-341.

6. Chisholm ST, Coaker G, Day B, Staskawicz BJ: Host-microbe interactions: shaping the evolution of the plant immune response. Cell 2006, 124(4):803-814.

7. Shan L, He P, Sheen J: Intercepting host MAPK signaling cascades by bacterial type III effectors. Cell Host Microbe 2007, 1(3):167.

8. Liu P, Yang Y, Pichersky E, Klessig DF: Altering expression of benzoic acid/ salicylic acid carboxyl methyltransferase 1 compromises systemic 
acquired resistance and PAMP-triggered immunity in arabidopsis. Mol Plant Microbe Interact 2010, 23(1):82-90

9. Robert-Seilaniantz A, Navarro L, Bari R, Jones JD: Pathological hormone imbalances. Curr Opin Plant Biol 2007, 10(4):372-379.

10. Bari $R$, Jones JDG: Role of plant hormones in plant defence responses. Plant Mol Biol 2009, 69(4):473-488.

11. Glazebrook J: Contrasting mechanisms of defense against biotrophic and necrotrophic pathogens. Annu Rev Phytopathol 2005, 43(1):205-227.

12. Grant M, Lamb C: Systemic immunity. Curr Opin Plant Biol 2006, 9(4):414-420.

13. Vlot AC, Dempsey D, Klessig DF, Vlot AC: Salicylic acid, a multifaceted hormone to combat disease. Annu Rev Phytopathol 2009, 47(1):177-206.

14. Ton J, Van Pelt J, Van Loon L, Pieterse C: Differential effectiveness of salicylate-dependent and jasmonate/ethylene-dependent induced resistance in Arabidopsis. Mol Plant Microbe Interact 2002, 15(1):27.

15. Ausubel FM, Katagiri F, Mindrinos M, Glazebrook J: Use of Arabidopsis thaliana defense-related mutants to dissect the plant response to pathogens. Proc Natl Acad Sci USA 1995, 92(10):4189-4196.

16. Alonso JM, Ecker JR: Moving forward in reverse: genetic technologies to enable genome-wide phenomic screens in Arabidopsis. Nat Rev Genet 2006, 7(7):524-536

17. Petersen LN, Ingle RA, Knight MR, Denby KJ: OXI1 protein kinase is required for plant immunity against Pseudomonas syringae in Arabidopsis. J Exp Bot 2009, 60(13):3727.

18. Volko SM, Boller T, Ausubel FM: Isolation of new Arabidopsis mutants with enhanced disease susceptibility to Pseudomonas syringae by direct screening. Genetics 1998, 149(2):537-548.

19. Glazebrook J, Ausubel FM: Isolation of phytoalexin-deficient mutants of Arabidopsis thaliana and characterization of their interactions with bacterial pathogens. Proc Natl Acad Sci USA 1994, 91(19):8955-8959.

20. Rogers EE, Ausubel FM: Arabidopsis enhanced disease susceptibility mutants exhibit enhanced susceptibility to several bacterial pathogens and alterations in PR-1 gene expression. Plant Cell 1997, 9(3):305-316.

21. Wiermer M, Feys BJ, Parker JE: Plant immunity: the EDS1 regulatory node. Curr Opin Plant Biol 2005, 8(4):383-389.

22. Schenk G, Elliott TW, Leung E, Carrington LE, Mitic N, Gahan LR, Guddat LW: Crystal structures of a purple acid phosphatase, representing different steps of this enzyme's catalytic cycle. BMC Struct Biol 2008, 8:6.

23. Mitić NN, Noble CJ, Gahan LR, Hanson GR, Schenk GG: Metal-ion mutagenesis: conversion of a purple acid phosphatase from sweet potato to a neutral phosphatase with the formation of an unprecedented catalytically competent $\mathrm{Mn}$ (II)Mn(II) active site. J Am Chem Soc 2009, 131(23):8173.

24. del Pozo JC, Allona I, Rubio V, Leyva A, de IP, Aragoncillo C, Paz-Ares J: A type 5 acid phosphatase gene from Arabidopsis thaliana is induced by phosphate starvation and by some other types of phosphate mobilising/ oxidative stress conditions. Plant J 1999, 19(5):579.

25. Zhang W, Gruszewski HA, Chevone BI, Nessler CL: An Arabidopsis Purple Acid Phosphatase with Phytase Activity Increases Foliar Ascorbate. Plant Physiol 2008, 146(2):431-440.

26. Liao H, Wong F, Phang T, Cheung M, Li WF, Shao G, Yan X, Lam H: GMPAP3, a novel purple acid phosphatase-like gene in soybean induced by $\mathrm{NaCl}$ stress but not phosphorus deficiency. Gene 2003, 318:103-111.

27. Kaida R, Satoh Y, Bulone V, Yamada Y, Kaku T, Hayashi T, Kaneko TS: Activation of beta-glucan synthases by wall-bound purple acid phosphatase in tobacco cells. Plant Physiol 2009, 150(4):1822-1830.

28. Ekrylander B, Flores M, Wendel M, Heinegard D, Andersson G: Dephosphorylation of Osteopontin and Bone Sialoprotein by Osteoclastic Tartrate-Resistant Acid-Phosphatase - Modulation of Osteoclast Adhesion In-Vitro. J Bio/ Chem 1994, 269(21):14853-14856.

29. Nuttleman P, Roberts RM: Transfer of iron from uteroferrin (purple acid phosphatase) to transferrin related to acid phosphatase activity. J Biol Chem 1990, 265(21):12192-12199.

30. Kaija H, Alatalo SL, Halleen JM, Lindqvist Y, Schneider G, Vaananen HK, Vihko $P$ : Phosphatase and oxygen radical-generating activities of mammalian purple acid phosphatase are functionally independent. Biochem Biophys Res Commun 2002, 292(1):128-132.

31. Hayman AR, Cox TM: Purple acid phosphatase of the human macrophage and osteoclast. Characterization, molecular properties, and crystallization of the recombinant di-iron-oxo protein secreted by baculovirus-infected insect cells. J Biol Chem 1994, 269(2):1294-1300.
32. Liang C, Tian J, Lam HM, Lim BL, Yan X, Liao H: Biochemical and molecular characterization of PVPAP3, a novel purple acid phosphatase isolated from common bean enhancing extracellular ATP utilization. Plant Physiol 2010, 152(2):854-865.

33. Li D, Zhu H, Liu K, Liu X, Leggewie G, Udvardi M, Wang D: Purple acid phosphatases of Arabidopsis thaliana comparative analysis and differential regulation by phosphate deprivation. J Biol Chem 2002, 277(31):27772-27781.

34. Kaffarnik FAR, Jones AME, Rathjen JP, Peck SC: Effector proteins of the bacterial pathogen Pseudomonas syringae alter the extracellular proteome of the host plant, Arabidopsis thaliana. Molecular \& cellular proteomics: MCP 2009, 8(1):145-156.

35. Winter D, Vinegar B, Nahal H, Ammar R, Wilson GV, Provart NJ: An "electronic fluorescent pictograph" browser for exploring and analyzing large-scale biological data sets. PLoS One 2007, 2(8):e718.

36. Zhu H, Qian W, Lu X, Li D, Liu X, Liu K, Wang D: Expression patterns of purple acid phosphatase genes in Arabidopsis organs and functional analysis of AtPAP23 predominantly transcribed in flower. Plant Mol Biol 2005, 59(4):581-594.

37. Tran HT, Qian W, Hurley BA, She Y, Wang D, Plaxton WC: Biochemical and molecular characterization of AtPAP12 and AtPAP26: the predominant purple acid phosphatase isozymes secreted by phosphate-starved Arabidopsis thaliana. Plant Cell Environ 2010, 33(11):1789-1803.

38. Dewdney J, Reuber TL, Wildermuth MC, Devoto A, Cui J, Stutius LM, Drummond EP, Ausubel FM: Three unique mutants of Arabidopsis identify eds loci required for limiting growth of a biotrophic fungal pathogen. Plant J 2000, 24(2):205-218.

39. Zipfel C, Robatzek S, Navarro L, Oakeley EJ, Jones JDG, Felix G, Boller T: Bacterial disease resistance in Arabidopsis through flagellin perception. Nature 2004, 428(6984):764-767.

40. Melotto $M$, Underwood W, Koczan J, Nomura K, He SY: Plant stomata function in innate immunity against bacterial invasion. Cell 2006, 126(5):969-980.

41. Menke FLH, Van Pelt JA, Pieterse CMJ, Klessig DF: Silencing of the mitogen-activated protein kinase MPK6 compromises disease resistance in Arabidopsis. Plant Cell 2004, 16(4):897-907.

42. Uquillas C, Letelier I, Blanco F, Jordana X, Holuigue L: NPR1-independent activation of immediate early salicylic acid-responsive genes in Arabidopsis. Mol Plant Microbe Interact 2004, 17(1):34-42.

43. Bozzo GG, Raghothama KG, Plaxton WC: Purification and characterization of two secreted purple acid phosphatase isozymes from phosphatestarved tomato (Lycopersicon esculentum) cell cultures. Eur J Biochem 2002, 269(24):6278-6286

44. Torres MA, Dangl JL: Functions of the respiratory burst oxidase in biotic interactions, abiotic stress and development. Curr Opin Plant Biol 2005, 8(4):397-403.

45. Ferrari S, Galletti R, Denoux C, De Lorenzo G, Ausubel FM, Dewdney J: Resistance to botrytis cinerea induced in Arabidopsis by elicitors Is independent of salicylic acid, ethylene, or jasmonate signaling but requires PHYTOALEXIN DEFICIENT. Plant Physiol 2007, 144(1):367-379.

46. Ferrari S, Plotnikova JM, De Lorenzo G, Ausubel FM: Arabidopsis local resistance to Botrytis cinerea involves salicylic acid and camalexin and requires EDS4 and PAD2, but not SID2, EDS5 or PAD4. Plant J 2003, 35(2):193-205.

47. Zhou N, Tootle TL, Tsui F, Klessig DF, Glazebrook J: PAD4 functions upstream from salicylic acid to control defense responses in Arabidopsis. Plant Cell 1998, 10(6):1021-1030.

48. Schenk G, Mitić N, Hanson GR, Comba P: Purple acid phosphatase: A journey into the function and mechanism of a colorful enzyme. Coord Chem Rev 2013, 257(2):473-482.

49. Schenk G, Guddat LW, Ge Y, Carrington LE, Hume DA, Hamilton S, de Jersey $\mathrm{J}$ : Identification of mammalian-like purple acid phosphatases in a wide range of plants. Gene 2000, 250(1-2):117-125.

50. Murashige T, Skoog F: A revised medium for rapid growth and bioassays with tobacco tissue cultures. Physiol Plant 1962, 15:473-497.

51. Alonso JM, Stepanova AN, Leisse TJ, Kim CJ, Chen H, Shinn P, Stevenson DK Zimmerman J, Barajas P, Cheuk R, Gadrinab C, Heller C, Jeske A, Koesema E, Meyers CC, Parker H, Prednis L, Ansari Y, Choy N: Genome-wide insertional mutagenesis of Arabidopsis thaliana. Science 2003, 301(5633):653.

52. Morcuende R, Bari R, Gibon Y, Zheng W, Pant BD, Bläsing O, Usadel B, Scheible W: Genome-wide reprogramming of metabolism and regulatory networks of Arabidopsis in response to phosphorus. Plant Cell Environ 2007, 30(1):85-112. 
53. Chomczynski P, Sacchi N: Single-step method of RNA isolation by acid guanidinium thiocyanate-phenol-chloroform extraction. Anal Biochem 1987, 162(1):156-159.

54. Torres MA, Dangl JL, Jones JDG: Arabidopsis gp91(phox) homologues AtrbohD and AtrbohF are required for accumulation of reactive oxygen intermediates in the plant defense response. Proc Natl Acad Sci USA 2002, 99(1):517-522.

doi:10.1186/1471-2229-13-107

Cite this article as: Ravichandran et al: Purple Acid Phosphatase 5 is

required for maintaining basal resistance against Pseudomonas syringae

in Arabidopsis. BMC Plant Biology 2013 13:107.

\section{Submit your next manuscript to BioMed Central and take full advantage of:}

- Convenient online submission

- Thorough peer review

- No space constraints or color figure charges

- Immediate publication on acceptance

- Inclusion in PubMed, CAS, Scopus and Google Scholar

- Research which is freely available for redistribution 